\title{
Adaptive MIMO Detection of OFDM Signals in an Underwater Acoustic Channel
}

\author{
Patricia Ceballos Carrascosa \\ Massachusetts Institute of Technology \\ Email: patriceb@mit.edu
}

\author{
Milica Stojanovic \\ Massachusetts Institute of Technology \\ Email: millitsa@mit.edu
}

\begin{abstract}
Frequency and time correlation of the underwater channel are exploited to obtain an adaptive channel estimation algorithm for MIMO spatial multiplexing with low complexity and low overhead. Non-uniform Doppler compensation is performed by extending the adaptive synchronization method [1] to multiple transmitters. Algorithm performance is demonstrated on experimental data recorded in several shallow water channels over distances on the order of $1 \mathrm{~km}$. Nearly error-free performance is observed for two and four transmitters with $\operatorname{BCH}(64,10)$ encoded QPSK signals. We report in detail on an experiment where an information rate of $23 \mathrm{kbps}(4$ transmitters $\times 0.75 \mathrm{sps} / \mathrm{Hz} /$ transmitter $\times 2$ bits $/$ symbol $\times 10 / 64 \times 24 \mathrm{kHz})$ is achieved using 2048 carriers in a $24 \mathrm{kHz}$ bandwidth.
\end{abstract}

\section{INTRODUCTION}

Orthogonal frequency division multiplexing (OFDM) has been used in underwater communications as an alternative to single carrier broadband modulation to achieve high data rate transmission [1]-[4]. It has proved to be an effective technique for combating the multipath delay spread without the need for complex time-domain equalizers.

Multiple transmit and receive antennas can be used to form multiple-input multiple-output (MIMO) channels to increase the system capacity. The combination of MIMO and OFDM is an appealing low-complexity solution for highly spectrally efficient communications over the bandwidth-limited frequencyselective underwater channels.

Previous work on MIMO underwater communications includes space-time coding to increase link reliability (an Alamouti design has been conceptually described and tested through simulation in [5]) and spatial multiplexing to increase the data rate $($ a $2 \times 4$ system was demonstrated using experimental data in [4]).

The most challenging task in a MIMO communication system is channel estimation. Because each received signal contains data from all the transmitters, multiple channels have to be estimated simultaneously. Research on channel estimation for wireless radio OFDM systems abounds, including methods such as [6] that compute the channel impulse response ( $L$ coefficients in the time domain) instead of the transfer function ( $K$ coefficients in the frequency domain). By doing so, the number of channel parameters is reduced when $L<K$, which is often the case, but this solution still requires the inversion of large matrices, whose size is proportional to $N_{t} L$, where $N_{t}$ is the number of transmit elements. Complexity can be reduced by designing optimal training signals and exploiting the correlation between consecutive OFDM blocks, an approach proposed in [7]. A different approach, also for two transmitters, was proposed in [8], where the channel is assumed to be constant over two adjacent carriers.

The problem of simultaneous estimation of multiple channels can also be decomposed into sequential estimation of individual channels by sending pilot tones from one transmitter at a time. In other words, while one transmitter is active, all the others remain silent. This approach was investigated in [4] and shown to provide good results in trials with experimental underwater acoustic data. However, its efficiency is limited to systems with a small number of transmitters (e.g. two). As the number of transmitters grows, so does the overhead ( $N_{t} L$ carriers per each transmitter), thus eventually destroying the original goal of using spatial multiplexing to increase the system capacity.

To address this problem, we focus on channel estimation that does not require inactive carriers, and makes use of symbol decisions to reduce the number of pilots. The aim of this paper is to present an adaptive channel estimation algorithm for MIMO underwater spatial multiplexing. The major difference between the wireless radio and acoustic OFDM systems is that the latter suffer from non-uniform frequency offset caused by the motion-induced Doppler effect. We extend the algorithm [1], which performs adaptive synchronization for underwater OFDM signals, to multiple transmitters and couple it with an adaptive MIMO channel estimator. Furthermore, we include sparsing of the channel impulse response which was shown to offer improved performance in singleinput multiple-output (SIMO) detection of underwater acoustic OFDM signals [3]. The effectiveness of the new algorithm is demonstrated through experimental data processing.

The paper is organized as follows. In Sec. II we introduce the notation and the receiver algorithm. Experimental data results are presented in Sec. III, and conclusions are summarized in Sec. IV. Finally, Sec. V contains the summary. 


\section{RECEIVER ALGORITHM}

\section{A. System and channel model}

We consider a system with $N_{t}$ transmit and $N_{r}$ receive elements. Each transmitter is sending an OFDM signal in $K$ subbands, where the input data stream at transmitter $t$ is serialto-parallel converted into $K$ streams $d_{k}^{t}(n), k=0, \ldots, K-1$. The symbol duration is $T$, and one OFDM block occupies an interval $T^{\prime}=T+T_{g}$, where $T_{g}$ is the guard time which is assumed to be the multipath spread. The total system bandwidth is $B$, and the carrier spacing is $\Delta f=1 / T=B / K$. The data symbols $d_{k}^{t}(n)$ are assumed to take values from an arbitrary PSK constellation. The symbol rate is $R=K /\left(T+T_{g}\right)$ symbols per second, per transmitter.

Following the approach of [1], the received signal after FFT demodulation is modeled as

$$
y_{k}^{r}(n)=\sum_{t=1}^{N_{t}} H_{k}^{t r}(n) d_{k}^{t}(n) e^{j \theta_{k}^{t}(n)}+z_{k}^{r}(n),
$$

where the indices $t, r, k, n$ refer to the transmitter, receiver, subband and time, respectively. The coefficient $H_{k}^{t r}(n)$ represents the transfer function of the channel between transmitter $t$ and receiver $r$, evaluated at frequency $f_{k}=f_{0}+k \Delta f$, during the $n$th OFDM block. The phase $\theta_{k}^{t}(n)$ represents the offset caused by the motion-induced Doppler effect, which is assumed to be equal for all receivers, but may differ between the transmitters. Specifically, the phase distortion is modeled as

$$
\theta_{k}^{t}(n)=\theta_{k}^{t}(n-1)+a^{t}(n) 2 \pi f_{k} T^{\prime}
$$

where $a^{t}(n)$ is the residual Doppler factor after initial signal resampling. It is assumed to be constant during one OFDM block, but may change from one block to another. Specifically, if $a^{\prime t}(n)=v^{t}(n) / c$ denotes the ratio of relative transmitterreceiver velocity to the speed of sound, i.e. the Doppler rate of the received signal, and initial resampling is performed over several blocks using an estimate $a^{\prime \prime}$, then the residual Doppler factor is $a^{t}(n)=\left(a^{\prime t}(n)-a^{\prime \prime}\right) /\left(1+a^{\prime t}(n)\right)$ [9]. The model (2) holds so long as the Doppler shift is much smaller than the carrier spacing, i.e. $a^{t}(n) f_{k}<<\Delta f, \forall k, n, t$. The residual inter-carrier interference is then treated as additional noise, contained in the term $z_{k}^{r}(n)$.

Let us now form the vectors

$$
\begin{aligned}
& \mathbf{y}_{k}(n)=\left[y_{k}^{1}(n), \cdots, y_{k}^{N_{r}}(n)\right]^{T} \\
& \mathbf{d}_{k}(n)=\left[d_{k}^{1}(n), \cdots, d_{k}^{N_{t}}(n)\right]^{T} \\
& \mathbf{z}_{k}(n)=\left[z_{k}^{1}(n), \cdots, z_{k}^{N_{r}}(n)\right]^{T}
\end{aligned}
$$

and the matrices

$$
\begin{aligned}
\mathbf{H}_{k}(n) & =\left[\begin{array}{ccc}
H_{k}^{11}(n) & \cdots & H_{k}^{N_{t} 1}(n) \\
\vdots & & \vdots \\
H_{k}^{1 N_{r}}(n) & \cdots & H_{k}^{N_{t} N_{r}}(n)
\end{array}\right] \\
\boldsymbol{\Theta}_{k}(n) & =\operatorname{diag}\left[e^{j \theta_{k}^{1}(n)}, \cdots, e^{j \theta_{k}^{N_{t}}(n)}\right] .
\end{aligned}
$$

Using this notation, we have that

$$
\mathbf{y}_{k}(n)=\mathbf{H}_{k}(n) \boldsymbol{\Theta}_{k}(n) \mathbf{d}_{k}(n)+\mathbf{z}_{k}(n) .
$$

\section{B. Data detection}

Given the channel matrix and the phases, the least squares (LS) estimate of the data symbols transmitted on the $k$ th carrier follows from the expression (8), and it is given by

$$
\hat{\mathbf{d}}_{k}(n)=\boldsymbol{\Theta}_{k}^{-1}(n)\left[\mathbf{H}_{k}^{\prime}(n) \mathbf{H}_{k}(n)\right]^{-1} \mathbf{H}_{k}^{\prime}(n) \mathbf{y}_{k}(n)
$$

where the prime denotes conjugate transpose. When the channel and the phase are not known, their estimates will be used instead of the true values. Data detection is performed by soft (or hard) decision decoding of the estimates (9).

\section{Channel estimation}

In each OFDM block, $N_{t} N_{r}$ channel coefficients have to be estimated for each subband. However, only $N_{r}$ observations of the received signal $y_{k}^{r}(n)$ are available for each $k$. To reduce the number of unknowns, we will exploit both the frequency and the time correlation of the channel.

1) Frequency correlation: Assuming that the channel transfer function for each transmitter-receiver pair is the same over $M_{f}$ adjacent carriers, ${ }^{1}$ we have that

$$
H_{k+i}^{t r}(n)=H_{k}^{t r}(n), i=1, \cdots, M_{f}-1 .
$$

Using this assumption, and defining the following quantities,

$$
\begin{gathered}
\check{\mathbf{y}}_{k}^{r}(n)=\left[y_{k}^{r}(n), \cdots, y_{k+M_{f}-1}^{r}(n)\right]^{T} \\
\check{\mathbf{z}}_{k}^{r}(n)=\left[z_{k}^{r}(n), \cdots, z_{k+M_{f}-1}^{r}(n)\right]^{T} \\
\mathbf{H}_{k}^{r}(n)=\left[H_{k}^{1 r}(n), \cdots, H_{k}^{N_{t} r}(n)\right]^{T} \\
\check{\mathbf{d}}_{k}(n)=\left[d_{k}^{1}(n) e^{j \theta_{k}^{1}(n)}, \cdots, d_{k}^{N_{t}}(n) e^{j \theta_{k}^{N_{t}}(n)}\right] \\
\check{\mathbf{D}}_{k}(n)=\left[\begin{array}{c}
\check{\mathbf{d}}_{k}(n) \\
\vdots \\
\check{\mathbf{d}}_{k+M_{f}-1}(n)
\end{array}\right]
\end{gathered}
$$

we can write

$$
\check{\mathbf{y}}_{k}^{r}(n)=\check{\mathbf{D}}_{k}(n) \mathbf{H}_{k}(n)+\check{\mathbf{z}}_{k}^{r}(n) .
$$

This model serves as a basis for channel estimation. Depending on the range of subbands $k$ for which the assumption (10) is made and the model (16) formed, we distinguish between two types of channel estimates: one based on a fixed-window, and another based on a sliding-window of subbands.

In the case of fixed-window estimation, the received signal observations are divided into $K / M_{f}$ groups of $M_{f}$ subbands, ${ }^{2}$ where $M_{f} \geq N_{t}$. Each group of subbands is now treated independently, and a single estimate is obtained for all the subbands in that group. In other words, the assumption (10) is made for $k=0, M_{f}, \ldots\left(K / M_{f}-1\right) M_{f}$, and the model (16) is applied for the same range of $k$.

\footnotetext{
${ }^{1}$ This approach is taken in [8] for two carriers.

${ }^{2}$ Without the loss of generality, we assume that $M_{f}$ is even, and that $K / M_{f}$ is an integer.
} 
Given the data symbols and the phases, the LS estimate of the channel at each receiving element is given by

$$
\hat{\mathbf{H}}_{k}^{r}(n)=\left[\check{\mathbf{D}}_{k}^{\prime}(n) \check{\mathbf{D}}_{k}(n)\right]^{-1} \check{\mathbf{D}}_{k}^{\prime}(n) \check{\mathbf{y}}_{k}^{r}(n)
$$

where $k=0, M_{f}, \ldots\left(K / M_{f}-1\right) M_{f}$. The remaining estimates are obtained using the assumption (10), i.e. $\hat{\mathbf{H}}_{k+i}^{r}(n)=$ $\hat{\mathbf{H}}_{k}^{r}(n)$ for $i=1, \ldots, M_{f}-1$.

When the data and the phases are not known, the symbol decisions and phase estimates will be used instead. Assuming that the channel does not change much during one OFDM block, the channel estimates from a previous block are used to make tentative symbol decisions that will in turn be used to update the phase and the current channel estimate. Note that pilot tones can also be used to aid decision-directed operation, but their number does not need to be constrained by the channel length as in [4].

In the case of sliding-window estimation, the first group of observations is defined as before, $y_{0}^{r}(n), y_{1}^{r}(n), \ldots y_{M_{f}-1}^{r}(n)$, and the initial channel estimate is obtained from it. Starting from here, each new group is defined by sliding the window of size $M_{f}$ by one, to compute the channel estimate at the next carrier. The modeling equation (16) remains the same, but the estimates are computed for all $k=0,1, \ldots K-M_{f}$. Specifically, we associate a channel estimate obtained from a group of observations with the mid frequency occupied by that group,

$$
\hat{\mathbf{H}}_{k+M_{f} / 2-1}^{r}(n)=\left[\check{\mathbf{D}}_{k}^{\prime}(n) \check{\mathbf{D}}_{k}(n)\right]^{-1} \check{\mathbf{D}}_{k}^{\prime}(n) \check{\mathbf{y}}_{k}^{r}(n)
$$

where $k=0,1, \ldots K-M_{f}$. The band-edge estimates are set to $\hat{\mathbf{H}}_{i}^{r}(n)=\hat{\mathbf{H}}_{M_{f} / 2-1}^{r}(n)$ for $i=0, \ldots M_{f} / 2-2$, and $\hat{\mathbf{H}}_{i}^{r}(n)=\hat{\mathbf{H}}_{K-M_{f} / 2-1}^{r}(n)$ for $i=K-M_{f} / 2, \ldots K-1$. The sliding-window method requires more computations, but gives better performance than the fixed-window method.

Fig. 1 illustrates the operation of the two methods for an example of $M_{f}=4$. For simplicity, a single receiving element is assumed. In the case of fixed-window estimation, the first four observations, $y_{0}(n), y_{1}(n), y_{2}(n), y_{3}(n)$, are used to form $\hat{H}_{0}(n)$. This estimate is used for all four subbands, i.e. $\hat{H}_{0}(n)=\hat{H}_{1}(n)=\hat{H}_{2}(n)=\hat{H}_{3}(n)$. The same principle is applied to the next four observations, $y_{4}(n), y_{5}(n), y_{6}(n), y_{7}(n)$, and so on. In the case of sliding-window estimation, the observations form the first four subbands are used to form $\hat{H}_{1}(n)$, which is extrapolated to all lower subbands, $\hat{H}_{0}(n)=$ $\hat{H}_{1}(n)$ in this case. The next estimate, $\hat{H}_{2}(n)$, is obtained by sliding the window to capture $y_{1}(n), y_{2}(n), y_{3}(n), y_{4}(n)$, and so on. The last estimate will be extrapolated to the remaining subbands at the high band-edge.

In order to carry out the channel estimation as described so far, the channel transfer function has to be assumed constant over $M_{f} \geq N_{t}$ adjacent carriers to provide a sufficient number of observations. Since the coherence frequency of the channel is given by the inverse of its multipath spread, $T_{m p}, M_{f}$ has to be chosen such that

$$
M_{f} \Delta f<<1 / T_{m p}
$$

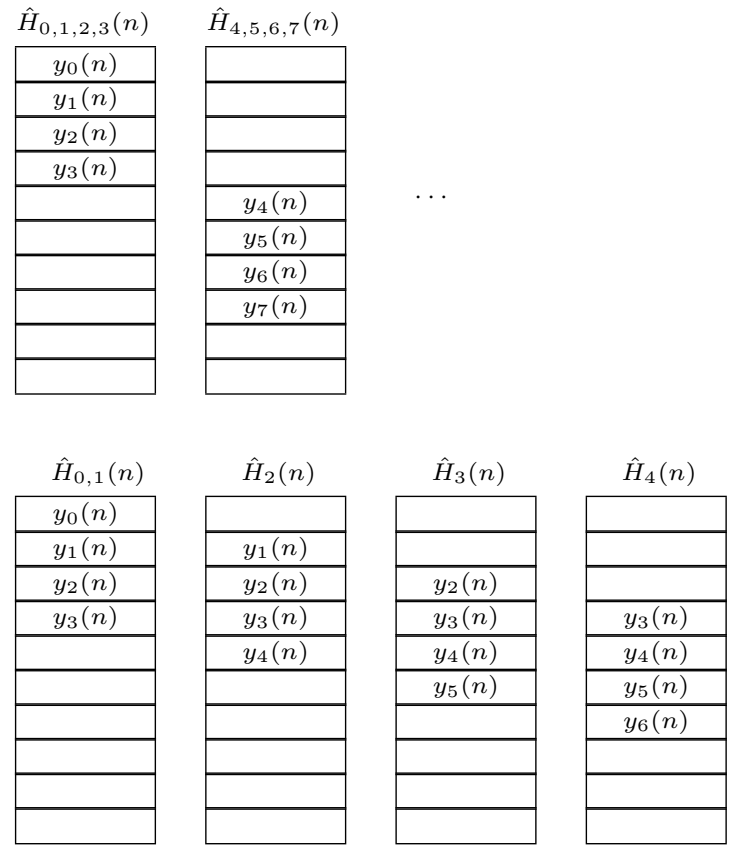

Fig. 1. Channel estimation using fixed-window (top) or sliding-window (bottom), with $M_{f}=4$.

This assumption is more easily justified when a greater number of carriers $K$ is used in a given bandwidth $B$, as the subband width $\Delta f=K / B$ is then narrower. A greater number of subbands also implies a greater bandwidth efficiency, defined (for each transmitter) as the ratio of the symbol rate $R$ to the occupied bandwidth $B$,

$$
\frac{R}{B}=\frac{K /\left(T+T_{g}\right)}{K / T}=\frac{1}{1+\alpha} .
$$

where $\alpha=T_{g} B / K$. Hence, it is advantageous to use large $K$, both from the viewpoint of maximizing the bandwidth efficiency, and from the viewpoint of satisfying the assumption (10).

The greatest number of subbands that can be used in a given system is limited by two factors: (1) the motion-induced Doppler shift, which accounts for the phase coherence, and (2) the temporal coherence of the channel (the Doppler spread corresponding to the inherent channel variation, regardless of the motion). Specifically, we recall that post-FFT processing is based on the assumption of small residual Doppler shift, i.e. $a^{t}(n) f_{k}<<\Delta f, \forall t, k, n$. If this condition is not satisfied, the signals will shift out of their allocated subbands, causing both a loss of phase coherence in the desired signal, and intercarrier interference (ICI). The basic model (8) will then no longer hold, and the simple post-FFT processing, which is based on it, will fail.

The second factor, namely the temporal channel coherence, refers to the fact that even in the absence of motion-induced Doppler distortion $\left(a^{t}(n)=0\right)$, the channel coefficients $H_{k}^{t r}(n)$ may be varying in time. Adaptive decision-directed receiver operation is based on the assumption that this variation 
is slow, so that the channel does not change much from one block to the next. The validity of such an assumption depends on the relationship between the inter-block separation $T^{\prime}$ and the coherence time of the channel, which is proportional to the inverse of its inherent Doppler spread, $B_{d}$. Clearly, in order for $T^{\prime}<<1 / B_{d}$ to hold, the block duration $T$ must be limited, i.e. for a fixed bandwidth $B$, the number of carriers $K=B T$ cannot exceed this coherence limit.

Ideally, $K$ will be large enough so that good bandwidth efficiency is achieved, while the corresponding $\Delta f$ is small enough for the assumption (19) to hold. If this is not the case, it will not be possible to exploit the frequency correlation (10) to obtain a sufficient number of observations for channel estimation. In such a case, one may resort to exploiting the time correlation between adjacent OFDM blocks.

2) Time correlation: Time correlation is exploited by assuming that the channel transfer function does not change between $M_{t}$ consecutive OFDM blocks, ${ }^{3}$ i.e.

$$
H_{k}^{t r}(n)=H_{k}^{t r}(n-m), m=1, \cdots, M_{t}-1 .
$$

The channel estimation problem can now be redefined to include this assumption in addition to the frequency correlation assumption (10). The number of observations needed for each estimate now has to be $M=M_{t} M_{f} \geq N_{t}$. As before, fixed-window (17) or sliding-window estimation (18) can be performed. The concept of a "window" now extends into the time domain. The sliding time window requires only one new block worth of data symbols (instead of $M_{t}$ ), and may be preferable over the fixed-window on a time-varying channel.

In order to exploit the time correlation of the channel by combining adjacent blocks, $M_{t}$ has to chosen such that

$$
M_{t} T^{\prime}<<1 / B_{d}
$$

where $T^{\prime}=T(1+\alpha)$, and $\alpha$ is the factor associated with the bandwidth efficiency (20).

Combining this condition with the frequency coherence requirement (19), we find that the total number of observations $M$ has to satisfy

$$
N_{t} \leq M<<\frac{1}{B_{d} T_{m p}} \cdot \frac{1}{1+\alpha} .
$$

This condition implies that regardless of $M$, there is a limit on the total number of transmit elements that can be used with low-complexity processing, and this limit depends on the coherence properties of the channel.

As far as the receiver design is concerned, there are several trade-offs in choosing the value of $M$, and, more specifically, the values of $M_{f}$ and $M_{t}$. In general, $M$ can be as low as $N_{t}$. This choice has the advantage of lowest computational complexity, as well as the least restrictive coherence requirements. However, it suffers most from the estimation noise, and is also associated with the highest incidence of singular data matrices (we will comment more on this issue when we discuss the experimental results). Consequently, it may be advantageous

\footnotetext{
${ }^{3}$ This approach is taken in [7] for two blocks.
}

to use $M$ somewhat greater than the minimum, e.g. $M=2 N_{t}$ (this choice provided uniformly good results with experimental signals).

Once the value of $M$ has been fixed, $M_{f}$ and $M_{t}$ should be determined in accordance with the frequency and time coherence requirements (19) and (22). These requirements must be weighed in light of the number of carriers $K$ used in an OFDM system with a given bandwidth. Specifically, they imply the following constraint:

$$
M_{f} B T_{m p}<<K<<\frac{B}{M_{t} B_{d}(1+\alpha)} .
$$

Recalling that bandwidth efficiency improves with $K$, we note that the preferred system design is one with $M_{t}$ as low as possible, e.g. $M_{t}=1$. Solutions with $M_{t}>1$ should be sought only when frequency coherence cannot be guaranteed over sufficiently many subbands (at least $N_{t}$ are needed for channel estimation), and carrier separation cannot be further reduced. Roughly speaking, for a small carrier separation $\Delta f$, more emphasis will be placed on exploiting the frequency correlation $\left(M_{f}>M_{t}\right)$, while for a large carrier separation, the emphasis will shift to exploiting the time correlation $\left(M_{t}>M_{f}\right)$. Note also that the interpretation of the "much less" sign is a soft one, and that system design ultimately has to be judged in a field test. We will comment more on these trade-offs when we discuss the particular examples in Sec.III.

\section{Adaptive filtering}

In order to improve the performance of the algorithm, time correlation is further exploited by adaptive filtering of the channel estimates. Furthermore, fewer than $K$ coefficients $\hat{h}_{l}^{t r}(n)$ may be needed to completely describe the channel, allowing for channel sparsing [3]. The complete algorithm description is beyond the scope of this paper, and deferred to a later full-length publication.

The complexity of the algorithm is dominated by matrix inversions, of which there are at most $K$ per OFDM block, each of size $N_{t} \times N_{t}$. Because $N_{t}$ is usually a small number, and because these operations can be carried out in parallel for different carriers, the overall complexity remains low compared to the methods such as [6], which require inversion of a single matrix for all carriers, but its size is $N_{t} L \times N_{t} L$, where $L$ is proportional to the total multipath spread of the channel, $L \sim B T_{m p}$.

\section{EXPERIMENTAL RESULTS}

The receiver algorithm has been tested in three different MIMO-OFDM experiments. Table I summarizes the salient features of the signals used in these experiments. All the data were collected by the Woods Hole Oceanographic Institution (WHOI).

Experiment 1 was part of the "AUV Fest," held at the Panama City Beach, FL, in June 2007. The transmitter and receiver were deployed about $9 \mathrm{~m}$ below the surface in $20 \mathrm{~m}$ deep water. The signals were sent from four equally spaced elements, with a total vertical aperture of $1 \mathrm{~m}$. The vertical 
TABLE I

EXPERIMENT SETTINGS.

\begin{tabular}{|l|l|l|l|l|l|l|l|l|}
\hline Experiment & $\mathbf{N}_{\mathbf{t}}$ & $\mathbf{N}_{\mathbf{r}}$ & Distance $[\mathbf{m}]$ & Frequency Band $[\mathbf{k H z}]$ & $\mathbf{K}$ & $\mathbf{T}[\mathbf{m s}]$ & $\mathbf{T}_{\mathbf{g}}[\mathbf{m s}]$ & $\mathbf{R} / \mathbf{B}=\frac{\mathbf{T}}{\mathbf{T}+\mathbf{T}_{\mathbf{g}}}$ \\
\hline $\mathbf{1}: \mathbf{F L}$ & 2,4 & 8 & $500,1000,1500$ & $24-48$ & $128-2048$ & $5.3-85.3$ & 25 & $0.175-0.773$ \\
\hline $\mathbf{2}: \mathbf{M A}$ & 2 & 6 & 600 & $75-137.5$ & $1024-4096$ & $16.4-65.5$ & 16 & $0.506-0.804$ \\
\hline 3 : RI & 2 & 12 & 400,1000 & $10-12.4$ & $128-256$ & $52.4-104$ & 15 & $0.777-0.874$ \\
\hline
\end{tabular}

receiving array was $2 \mathrm{~m}$ in aperture with 8 elements equally spaced at $25 \mathrm{~cm}$. The center frequency was $36 \mathrm{kHz}$, with a $24 \mathrm{kHz}$ bandwidth. The sampling rate was $96 \mathrm{kHz}$.

In this experiment, $N_{d}=32768$ QPSK symbols per transmitter were sent from either two or four transmitters (chosen as maximally spaced), using a varying number of subbands ranging from 128 to 2048 . The guard interval was chosen to be $T_{g}=25 \mathrm{~ms}$ with zero-padding (ZP). The signals were coded using the $\mathrm{BCH}(64,10)$ code. This code was selected so as to match the one currently implemented in the WHOI modem. Each string of 10 bits was encoded into a 32-symbol QPSK codeword, until $K$ symbols were obtained. These symbols were then assigned to the OFDM carriers in such a manner that the symbols of one codeword were maximally separated in frequency. Soft decision decoding was employed.

Fig. 2 shows the received signal from a frame with four transmitters and 1024 carriers. The frame preamble is a PN sequence of length 127, quadrature modulated at $24 \mathrm{ksps}$ using the center frequency of $36 \mathrm{kHz}$. Frame synchronization is performed by matched filtering to the preamble.

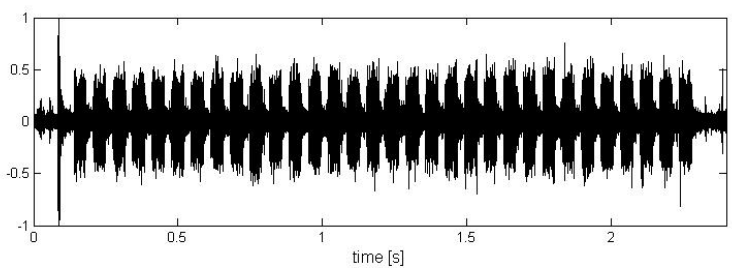

Fig. 2. Received signal in experiment 1 , for a $K=1024$ frame with four transmitters at transmission distance of $1500 \mathrm{~m}$. Shown are the preamble and $N_{d} / K=32$ OFDM blocks.

Channel estimates for this frame are shown in Fig. 3. Channel estimation is performed for all transmitter-receiver pairs using the sliding-window technique. Shown are the results for all transmitting elements, the top and the bottom receiving element. From the 1024 coefficients that are computed for each transmitter-receiver pair, only between 9 and 30 are preserved after channel sparsing ( $15 \mathrm{~dB}$ below the maximal amplitude). The different peaks in the channel estimates can be associated with multiple surface and bottom reflections calculated from the geometry of the experiment.

The phase estimates are shown in Fig. 4. The estimated Doppler factors $\hat{a}^{t}(n)$ range between $-5 \cdot 10^{-5}$ and $5 \cdot 10^{-5}$. It is interesting to note that although the transmit elements are close together, their phases may differ significantly. This behavior can be explained by the fact that each transmitter's relative motion with respect to the receiver array may be different. Individual phase estimation for each transmitter
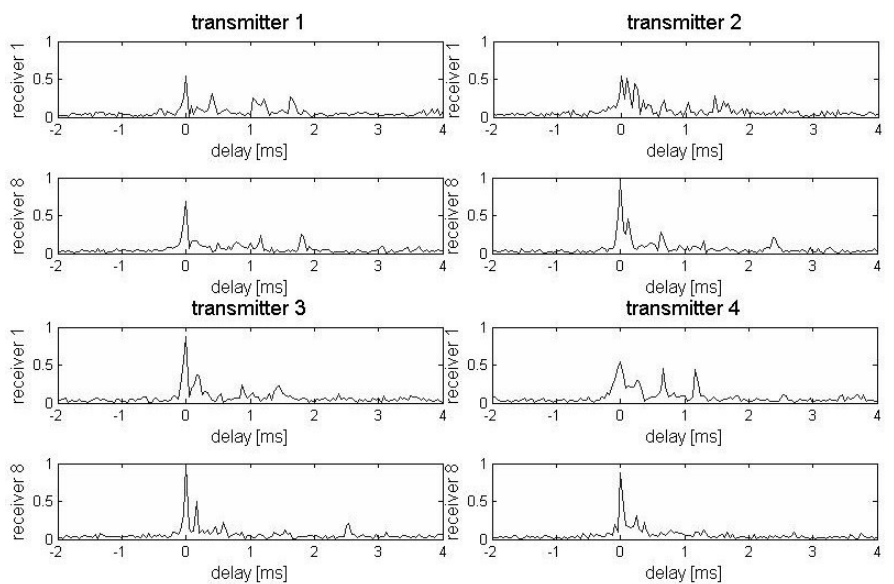

Fig. 3. Channel estimates (magnitude of the impulse response) for a frame with $K=1024$ carriers and four transmitters at transmission distance of $1500 \mathrm{~m}$.

proved to be crucial for successful data detection in this experiment.
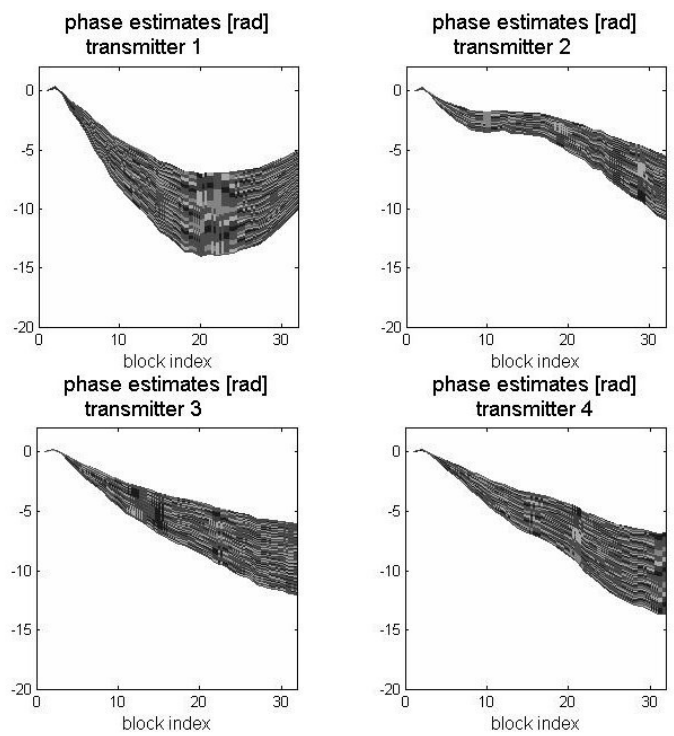

Fig. 4. Phase estimates for all carriers (non-uniform compensation) for a frame with $K=1024$ at transmission distance of $1500 \mathrm{~m}$.

Table II provides the summary of algorithm performance with two transmitters. A total of $M=2 N_{t}=4$ observations were used, and various combinations of $M_{f}$ and $M_{t}$ were investigated for each value of $K$. Listed in the table are those combinations that provided the best performance. As expected, 
$M_{t} \times M_{f}=M \times 1$ was the best choice for the lowest value of $K$, shifting to $1 \times M$ for higher values of $K$ and narrower carrier separations $\Delta f$. The results provided are for one frame of data sent $\left(N_{d}=32768\right.$ QPSK symbols per transmitter) with $N_{p}=K / 32$ pilots. No degradation in performance was observed without the use of pilot tones, except for $K=2048$. The BER indicated in the table represents the ratio of erroneously decoded bits to the total number of bits transmitted, averaged over all transmitters. We observe that excellent results are achieved in this experiment. BER on the order of $5 \cdot 10^{-2}$ is obtained without coding, indicating that a lower rate code could be used in these conditions. We also note that the the guard time of $25 \mathrm{~ms}$ was unnecessarily long; $5 \mathrm{~ms}$ would have sufficed for this channel, yielding a higher bandwidth efficiency.

TABLE II

EXPERIMENT 1: RESULTS FOR SEVERAL MIMO CONFIGURATIONS WITH TWO TRANSMITTERS.

\begin{tabular}{|l|l|l|l|l|l|}
\hline $\mathbf{K}$ & $\mathbf{1 2 8}$ & $\mathbf{2 5 6}$ & $\mathbf{5 1 2}$ & $\mathbf{1 0 2 4}$ & $\mathbf{2 0 4 8}$ \\
\hline $\mathbf{\Delta f}[\mathrm{Hz}]$ & 187.5 & 93.75 & 46.88 & 23.44 & 11.72 \\
\hline $\mathbf{T}[\mathrm{ms}]$ & 5.3 & 10.7 & 21.3 & 42.7 & 85.3 \\
\hline $\mathbf{M}_{\mathbf{t}} \times \mathbf{M}_{\mathbf{f}}$ & $4 \times 1$ & $2 \times 2$ & $1 \times 4$ & $1 \times 4$ & $1 \times 4$ \\
\hline $\mathbf{B E R}(500 \mathrm{~m})$ & 0 & 0 & 0 & 0 & 0 \\
\hline $\mathbf{B E R}(1000 \mathrm{~m})$ & 0 & 0 & 0 & 0 & 0 \\
\hline $\mathbf{B E R}(1500 \mathrm{~m})$ & 0 & 0 & 0 & 0 & 0 \\
\hline
\end{tabular}

Performance of the receiver for $K=1024$ carriers at a transmission distance of $1000 \mathrm{~m}$ is detailed in Fig. 5, which shows the scatter plot of the symbol estimates and the corresponding mean squared error over time and carriers. Shown also are the phase estimates, the Doppler factor, and the impulse response estimate before sparsing.
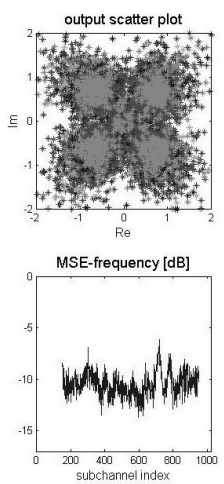
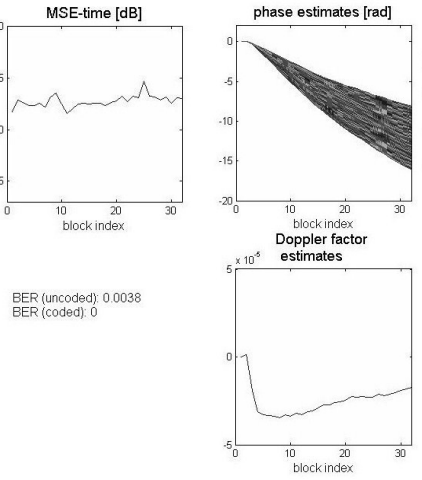

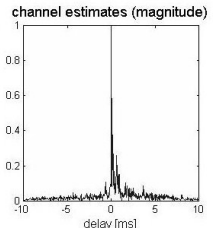

delay [ms]
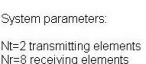

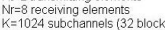
chennel sparsing threshold 0.2 no overlap-and-add no pilct tones
channel tractong paramiter 0.1 16 significant coefficient
per channel estimate

Fig. 5. Signal processing results for experiment 1 at transmission distance of $1000 \mathrm{~m}$. Band-edge carriers were disabled to avoid the transducer cut-off.

Results for four transmitters are summarized in Table III. A total of $M=2 N_{t}=8$ observations were used in this case. As before, the results refer to one frame of data sent $\left(N_{d}=\right.$ 32768 QPSK symbols per transmitter) with $N_{p}=K / 32$ pilots per transmitter. The BER indicated in the table is that after decoding; without coding, the BER is on the order of 0.15 .

We observe that $K=128$ is a poor design choice in this case, most likely because it challenges the frequency coherence assumption (19). It is also interesting to note that performance tends to improve with transmission distance, which can be explained by an increasing coherence frequency (decreasing multipath spread). For example, if the system geometry is used to compute the delay spread of the surface-bottom-surface reflection $(2.2 \mathrm{~ms}, 1.1 \mathrm{~ms}$ and $0.75 \mathrm{~ms})$, the corresponding coherence frequency is found to be $450 \mathrm{~Hz}, 900 \mathrm{~Hz}$ and $1350 \mathrm{~Hz}$ for the transmission distance of $500 \mathrm{~m}, 1000 \mathrm{~m}$ and $1500 \mathrm{~m}$, respectively. Hence, the frequency coherence assumption is more easily justified at longer ranges. These numbers also support the fact that performance improves for higher values of $K$. Notably, for $K=512,1024$ and $2048, M_{f} \Delta f=93.75 \mathrm{~Hz}$, and, at least for $1500 \mathrm{~m}$, frequency coherence can be assumed. It may be worth noting that at this distance, $K=512$ and 1024 offered equally good performance without pilot assistance.

TABLE III

EXPERIMENT 1: RESULTS FOR SEVERAL MIMO CONFIGURATIONS WITH FOUR TRANSMITTERS

\begin{tabular}{|l|l|l|l|l|l|}
\hline $\mathbf{K}$ & $\mathbf{1 2 8}$ & $\mathbf{2 5 6}$ & $\mathbf{5 1 2}$ & $\mathbf{1 0 2 4}$ & $\mathbf{2 0 4 8}$ \\
\hline $\mathbf{\Delta f}[H z]$ & 187.5 & 93.75 & 46.88 & 23.44 & 11.72 \\
\hline $\mathbf{T}[m s]$ & 5.3 & 10.7 & 21.3 & 42.7 & 85.3 \\
\hline $\mathbf{M}_{\mathbf{t}} \times \mathbf{M}_{\mathbf{f}}$ & $8 \times 1$ & $4 \times 2$ & $4 \times 2$ & $2 \times 4$ & $1 \times 8$ \\
\hline $\begin{array}{l}\text { BER } \\
(500 \mathrm{~m})\end{array}$ & 0.1 & $4 \cdot 10^{-3}$ & $4 \cdot 10^{-3}$ & $10^{-3}$ & $4 \cdot 10^{-3}$ \\
\hline $\begin{array}{l}\text { BER } \\
(1000 \mathrm{~m})\end{array}$ & 0.2 & $10^{-3}$ & $8 \cdot 10^{-4}$ & $2 \cdot 10^{-3}$ & $9 \cdot 10^{-3}$ \\
\hline $\begin{array}{l}\text { BER } \\
(1500 \mathrm{~m})\end{array}$ & $3 \cdot 10^{-3}$ & $10^{-3}$ & 0 & 0 & 0 \\
\hline
\end{tabular}

For both two and four transmitters, at least under the conditions of the present experiment and for the bandwidth used, the best performance of the algorithm was observed with 512 and 1024 carriers. These design choices resulted in consistently good performance, monitored over multiple signal frames. As $K$ further increases, the limit imposed by the phase coherence of the channel is occasionally reached, causing a failure with $K=2048$, particularly at shorter distances.

While the above results were obtained using $M=2 N_{t}$ observations, good performance was also achieved with $M=$ $N_{t}$ observations, which further reduces the computational complexity. However, as the size of the matrix $\check{\mathbf{D}}_{k}(n)$ is reduced, its chances of having rank less than $N_{t}$ increase. This matrix depends on the random values of the data symbols in a current block, and, hence, it can happen so that its inverse, or pseudo-inverse for $M>N_{t}$, does not exist. In such a case, the new channel estimate cannot be computed, and the previous channel estimate is kept instead. This simple method provided excellent results in the present experiment.

Sliding-window estimation has been used both in time and in frequency. In order to reduce the complexity, fixed-window can be used in frequency without performance degradation, except with $M_{t} \times M_{f}=1 \times 8$. When operating in a decision-directed mode, sliding-window in time was found to be necessary.

Satisfactory results have also been observed for the other two experiments listed in Table I. In experiment 2, a very high frequency (VHF) transducer was used, yielding a raw bit rate of up to $203 \mathrm{kbps}$ (31.4 kbps after coding and with 
$N_{p}=K / 32$ ). It may be worth noting that in this experiment it sufficed to use a single phase estimate for both transmitters.

Experiment 3 was conducted as part of a larger experiment called "RACE'08." Error-free performance was obtained with 128 and 256 carriers, regardless of the selection of $M_{f}$ and $M_{t}(M=4)$, QPSK signals. Furthermore the uncoded BER was below 0.1 , allowing the receiver to operate in decisiondirected mode even without the decoded in the loop. In this experiment, 8-PSK signals were also used, resulting in errorfree performance with $K=256$ and $M_{t} \times M_{f}=1 \times 4$; however, coding was necessary to maintain decision-directed operation in this case.

\section{CONCLUSIONS}

The algorithm performance was demonstrated using experimental data from three collection sites, all corresponding to shallow water channels with a range on the order of $1 \mathrm{~km}$, but different frequency bands. The results obtained indicate that the optimal choice of system parameters varies with each experimental setting, and should be chosen accordingly. Nonetheless, the algorithm proposed is relatively simple, and general rules can easily be established for selecting its parameters $M_{t}$ and $M_{f}$. Non-uniform phase estimation is essential for enabling the decision-directed operation. When implemented in a MIMO configuration, an independent phase estimate should be associated with each transmitter to take into account the possibility of different Doppler effect.

As for the MIMO channel estimation, the number of observations needed to estimate the channel coefficients is at least $M=N_{t}$, while $M=2 N_{t}$ sufficed in all the experiments in our study. We found that fixed-window can be used in frequency to reduce the computational complexity in the majority of the cases studied, while sliding-window has to be used in time. Adaptive filtering further improves the channel estimates and is not overly sensitive to the choice of filter memory. Sparsing of the impulse response provided performance improvement with the same truncation threshold in all cases ( $15 \mathrm{~dB}$ below the maximal amplitude). Finally, the general system design should target the largest number of carriers $K$ for which temporal coherence can be maintained. With the $\mathrm{BCH}(64,10)$ code, nearly error-free performance was observed consistently in all experiments. The attendant bit rates after coding were $22.5 \mathrm{kbps}$ (four transmitters and 2048 carriers in $24 \mathrm{kHz}$ bandwidth), $31.4 \mathrm{kbps}$ (two transmitters and 4096 carriers in $62.5 \mathrm{kHz}$ bandwidth), and $2 \mathrm{kbps}$ (two transmitters and 256 carriers in $2.4 \mathrm{kHz}$ bandwidth, 8PSK).

\section{SUMMARY}

Multiple-input multiple-output (MIMO) communications were considered as a means of spatial multiplexing to increase the data rate supported by a band-limited underwater acoustic channel. The choice of OFDM as a modulation technique renders the signal in each subband free of intersymol interference, thus simplifying the problem of MIMO channel estimation.

The algorithm proposed incorporates compensation of the motion-induced non-uniform Doppler frequency offset across the wide OFDM signal bandwidth, adaptive MIMO channel estimation, and data detection. Channel estimation capitalizes on the frequency correlation between adjacent subbands and time correlation between adjacent OFDM blocks. By doing so, a sufficient number $M \geq N_{t}$ of received signal observations are provided for estimating the channel transfer function between each of the $N_{t}$ transmit and $N_{r}$ receive elements. In this low-complexity approach, a single matrix inversion of size $N_{t} \times N_{t}$ is required per carrier, and these operations can be performed in parallel for the $K$ carriers. The estimated frequency-domain (transfer function) coefficients are transformed into time-domain (impulse response) coefficients, where magnitude truncation is performed to account for the fact that an underwater channel is often sparse, and can thus be fully represented with fewer than $K$ coefficients. Sparsing of the impulse response eliminates the unnecessary noise, and yields improved performance. Adaptive filtering of the channel estimate is employed to further exploit the temporal coherence of the channel. Phase prediction, based on adaptive tracking of the Doppler factor, enables decision-directed operation, which, in turn, yields reliable tentative decisions for channel estimation, resulting in a significant reduction of the pilot overhead. Details of the adaptive MIMO channel estimation and phase prediction algorithm are deferred to a full-length publication.

\section{ACKNOWLEDGMENTS}

The authors would like to thank the WHOI acoustic communications group for conducting at-sea experiments. This work was supported in part by the ONR grants N00014-07-1-0202 and MURI N00014-07-1-0738.

\section{REFERENCES}

[1] M.Stojanovic, "Low complexity OFDM detector for underwater acoustic channels," IEEE Oceans Conf., September 2006.

[2] B.Li, S.Zhou, M.Stojanovic, L.Freitag, and P.Willett, "Multicarrier communication over underwater acoustic channels with nonuniform doppler shifts, "IEEE Journal of Oceanic Engineering, to appear 2008.

[3] M.Stojanovic, "OFDM for underwater acoustic communications: adaptive synchronization and sparse channel estimation," in Proc. ICASSP, 2008. OFDM over fast-varying underwater acoustic channels," IEEE Oceans Conf., June 2007.

[4] B.Li, S.Zhou, M.Stojanovic, L.Freitag, J.Huand, and P.Willett, "MIMO-OFDM over an underwater acoustic channel," in Proc. IEEE Oceans Conf., October 2007.

[5] R.F.Ormondroyd, "A robust underwater acoustic communication system using OFDM-MIMO," Oceans 2007- Europe, June 2007.

[6] Y.(G.)Li, N.Seshadri, and S.Ariyavisitakul, "Channel estimation for OFDM systems with transmitter diversity in mobile wireless channels," IEEE Journal on Selected Areas in Communications, Vol.17, No.3, pp.461-471, March 1999.

[7] Y.(G.)Li, "Simplified channel estimation for OFDM systems with multiple transmit antennas," IEEE Transactions on Wireless Communications, Vol.1, No.1, pp.67-75, January 2002.

[8] H.Minn, D.Kim, and V.(K.) Bhargava, "A reduced complexity channel estimation for OFDM systems with transmit diversity in mobile wireless channels," IEEE Transactions on Communications, Vol.50, No.5, pp.799-807, May 2002.

[9] M.Stojanovic, "Underwater acoustic communications: design considerations on the physical layer," The Fifth Annual Conference on Wireless on Demand Network Systems and Services (WONS 2008), pp.1-10, January 2008 . 